\title{
Cultivo en suspensión de Mytella guyanensis (Bivalvia:Mytilidae) en Isla Chira, Costa Rica: implicaciones ambientales y biológicas
}

\author{
Paul Ureña Juárez ${ }^{1}$, Christian Diaz Peralta²
}

1. Universidad Estatal a Distancia, Escuela de Ciencias Exactas y Naturales, Programa de Laboratorio, 474-2050 Sabanilla, San José, Costa Rica; purena@uned.ac.cr

2. Universidad Católica de la Santísima Concepción, Centro de Investigación en Biodiversidad y Ambientes Sustentables (CIBAS), Avda. Alonso de Ribera 2850, Cód. Postal 4090541, Concepción, Chile; chdiaz@ucsc.cl

Recibido: 30 de junio de $2020 \quad$ Aceptado: 22 de octubre de 2020

\section{Resumen}

La maricultura artesanal surge en las últimas décadas como una actividad productiva para el desarrollo socioeconómico de las islas del Golfo de Nicoya en Costa Rica. Determinar las condiciones ambientales y biológicas para impulsar el cultivo de Mytella guyanensis representa un gran insumo para el manejo y la comercialización de estos bivalvos de forma sostenible. Se evaluó el crecimiento, la mortalidad y su relación con los parámetros ambientales en un medio de cultivo en suspensión en Isla Chira. Durante 6 meses (octubre 2018-marzo 2019), se registraron los principales parámetros ambientales del medio de cultivo, se realizaron biometrías a 300 individuos de la población cultivada y, además, se estimó la tasa de mortalidad mediante el procesamiento de 60 canastas de mejillón. En términos generales, a) los parámetros físico-químicos monitoreados presentaron condiciones aptas para el cultivo de estos organismos, b) se registró una tasa de mortalidad del mejillón mensual promedio de $13 \% \pm 4,5$; y c) M. guyanensis alcanzó un tamaño apto para su comercialización y consumo ( $\geq 40 \mathrm{~mm}$ de longitud) a partir del cuarto mes de cultivo. El crecimiento de $M$. guyanensis mediante el sistema de producción en canastas suspendidas, tiende a suceder con mayor rapidez con respecto a su crecimiento en bancos naturales. El futuro de la Maricultura, dependerá en gran medida del seguimiento de los parámetros físico-químicos, de la caracterización de rangos óptimos para las especies cultivadas, como también, de la identificación de nuevos sitios aptos para cultivar Palabras clave: Maricultura, Bivalvo, Chora, Mitílidos, Parámetros físico-químicos

\begin{abstract}
In Costa Rica, the mariculture perspective has been increasing in the last decade as a strategy to mitigate social, environmental and economic conflicts within coastal communities, due to the misuse of marine resources from the locals. Diversifying the commercial offer and addressing a sustainable use of seafood in Isla Chira are the aims of this activity. Were to evaluate the growth, mortality rate and their relationship with environmental factors and conditions of the Mytella guyanensis suspended - culture broth. Monthly recording of the main environmental parameters of the culture broth took place during six months in addition to the biometric analysis of 300 individuals from the cultivated population in order to determine the appropriate commercial size. The mortality rate estimation was carried out using 60 baskets of organisms. The general environmental factor are suitable for the growing of the inoculated mussels. b) The average mussel's mortality rate was $13 \pm 4,5 \%$; c) mussel growth in baskets is greater than the growth in natural banks and finally d) $M$. guyanensis reaches a suitable size for commercialization and consumption from the fifth month of cultivation ( $\geq 40 \mathrm{~mm}$ in length). We failed to stablish a correlation among the physicochemical parameters from the environment and the mortality rate recorded for harvested mussels $(P>0,05)$. The future of mariculture will depend to a great extent on the monitoring of physical-chemical parameters, on the characterization of optimal ranges for cultivated species, as well as on the identification of new sites suitable for cultivation
\end{abstract}

Keywords: Mariculture, Bivalve, Chora, Mitilides, Physical-chemical parameters 


\section{Introducción}

La conservación y utilización racional de los océanos, los mares y los recursos marinos, constituye uno de los principales Objetivos de Desarrollo Sostenible (ODS), según la Agenda 2030 para el Desarrollo Sostenible de las Naciones Unidas. Para poder alcanzar una seguridad alimentaria, beneficios sociales, ambientales y económicos de una forma sostenible en el mundo, debemos de volver la mirada a las actividades pesqueras y a la acuicultura de pequeña y mediana escala. La acuicultura, en comparación con otros sectores productivos, ha tenido un repunte en su crecimiento en las últimas décadas, especialmente en países africanos y asiáticos (FAO, 2018). Esta práctica ha tomado gran relevancia en las comunidades costeras, ya que logra dotar a las personas, a través del uso de recursos naturales, de fuentes de empleo, nuevas oportunidades para comercializar sus productos, todo esto se va sumando para minimizar los índices de la pobreza mundial (FAO, 2018). Los moluscos son, por lo general, fauna muy común en ecosistemas marino-costeros, además son apreciados comercialmente por su alto valor proteínico, su riqueza en minerales y en vitaminas los convierte de gran interés nutricional para la salud humana (Astorga et al., 2007; Fuentes et al., 2009).

Una amplia cantidad de especies de mejillones son cultivadas con fines de comercialización a nivel mundial (Colombo et al., 2016). Es desde el Golfo de Nicoya en Costa Rica donde se produce la mayor cantidad de recursos pesqueros que abastece a diferentes sectores del país, destacan los pescadores y los maricultores artesanales de Isla Chira que tienen acceso a nula o poca tecnología para la producción y comercialización de estos recursos (Ross et al., 2014). En Costa Rica el estudio formal del cultivo de mejillones da inicio a finales de la década de los 80 `s, en bancos lodosos donde se encuentran naturalmente (Sibaja, 1985a, 1985b; Sibaja, 1986). Posteriormente, se han llevado a cabo estudios preliminares en cuanto a su cultivo en balsas, ciclos reproductivos, biometrías (Cruz \& Villalobos, 1993a, 1993b; Arroyo \& Marín, 1998) y recientemente la evaluación de sitios óptimos para el cultivo de bivalvos en el Golfo de Nicoya (Quesada, 2018).

Según los autores Timmons, Ebeling y Piedrahita (2009), hay factores ambientales como la temperatura, oxígeno disuelto, batimetría, salinidad entre otros, que inciden directamente en el crecimiento y supervivencia de los bivalvos. Los parámetros físicos químicos del agua marina, suelen tener variaciones considerables en la época lluviosa en la parte interna del Golfo de Nicoya. Se han reportado valores por debajo de los 20 ppm (Arroyo \& Marín, 1998). Bolaños (1988), registró niveles altos de turbidez en algunos sectores de Isla Chira $(0,53 \mathrm{~m})$ producto de la sedimentación en el medio, lo cual podría afectar directamente la filtración de alimentos por parte del mejillón, limitando su crecimiento.

Existe una tendencia a que Mytella guyanensis crezca con mayor prontitud en cultivos manejados (artificiales), con respecto a su crecimiento en bancos naturales (Carvajal, 1969). Leighton (1979), destaca que los mejillones en cultivo cuentan con un mejor acceso al recurso alimenticio en la columna de agua y por periodos más largos de tiempo. Las tasas de crecimiento de $M$. guyanensis alrededor del mundo tienden a ser muy variadas, debido a las condiciones ambientales particulares de cada zona. Sin embargo, los Mitílidos de países tropicales suelen presentar niveles de crecimiento mayores con respecto a especies de climas más templados (Mesas \& Tarifeño, 2015). El monitoreo de las condiciones ambientales y parámetros físicoquímicos del medio, constituyen indicadores para el cultivo comercial exitoso de mejillones y otros bivalvos (Diarte, Escamilla, De la Cruz, Granados \& Álvarez, 2013; Quesada, 2018).

Actualmente se desarrolla un Proyecto de cultivo de mejillón en Puerto Palito, Isla Chira, 
liderado por la Asociación Salvemos al Golfo de Nicoya (ASOSAGONY) (ver Figura 1). El presente estudio tiene como objetivo evaluar el crecimiento y la mortalidad del mejillón $(M$. guyanensis) cultivado en este proyecto por medio de canastas en suspensión, y su posible relación con los parámetros ambientales del medio de cultivo.

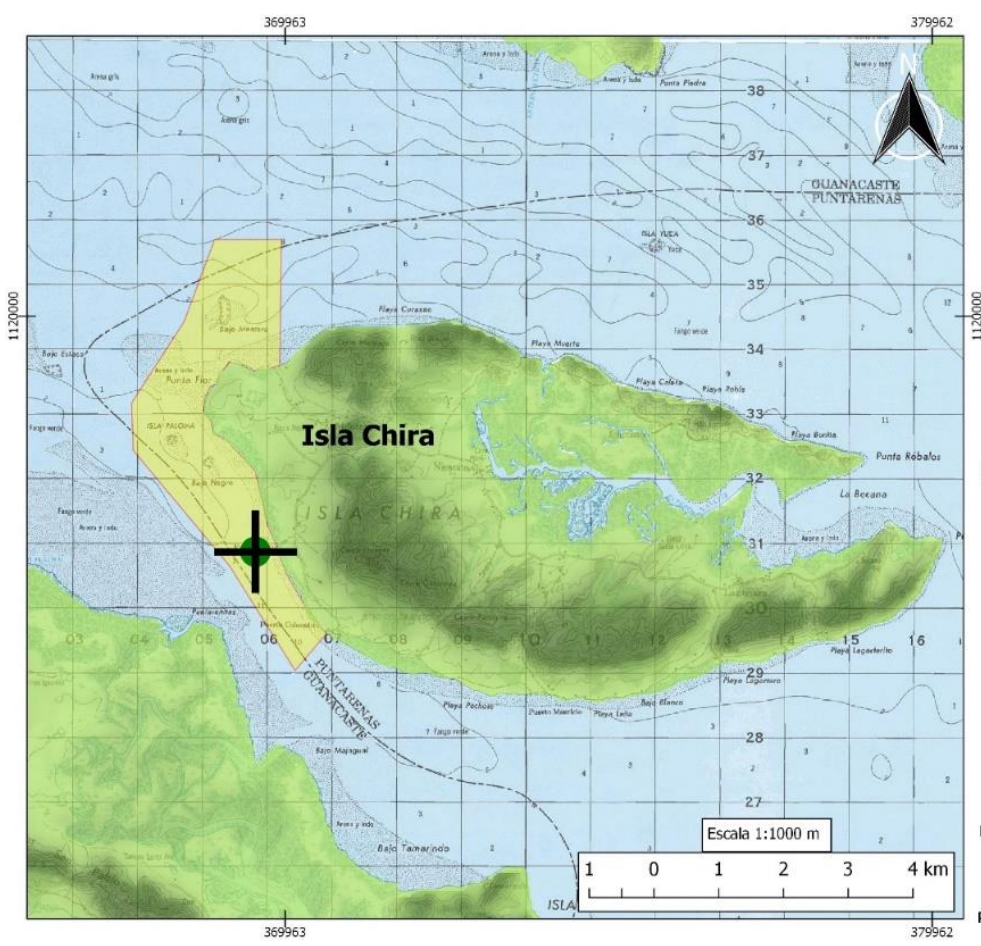

\section{Mapa de proyecto del Cultivo de Mejillón}

\section{Simbología}

Área de pesca responsable 201

-Ubicación de cultivo de mejillón
Lat $10.092940^{\circ}$ Lat $10.092940^{\circ}$

Diagrama de ubicación
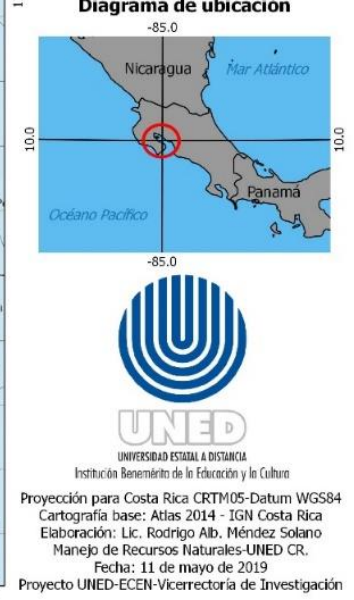

Figura 1. Ubicación del proyecto de Maricultura en la comunidad de Palito, Isla Chira. Fuente: Méndez, R. (2019).

\section{Materiales y Métodos}

Durante seis meses continuos (oct2018-mar2019) se visitó el proyecto de Mejillón en la comunidad de Puerto Palito, ubicada en el sector Oeste de Isla Chira, Puntarenas, Costa Rica $\left(10^{0} 09^{\prime} 31.9^{\prime}{ }^{\prime} \mathrm{N}\right.$, $\left.85^{\circ} 18^{\prime} 93.0^{`} \mathrm{~W}\right)$. El sistema de cultivo en suspensión se estableció mediante una estructura o plataforma flotante de madera de $3 \mathrm{~m}$ x $3 \mathrm{~m}$. El sistema de flotación, conocida como "balsa mejillonera" está conformado por 4 cilindros plásticos sellados, de un volumen de 273 litros cada uno (ver Figura 2). Se encuentran instalados a $300 \mathrm{~m}$ al Oeste de la comunidad de Palito. Cada una de las cuatro balsas instaladas, cuenta con aproximadamente 150 canastas elaboradas de malla de polipropileno, selladas con nylon (20cm de diámetro x $40 \mathrm{~cm}$ de alto), para un total de 600 canastas. Estas canastas son reutilizadas en cosechas posteriores, una vez que el mejillón alcanza su talla comercial ( $\geq 40 \mathrm{~mm}$ ). Cabe indicar, que los individuos jóvenes que se seleccionaron para iniciar la colonia se tomaron de colectores artificiales plásticos, que también se encuentran en suspensión (ver Figura 3). 


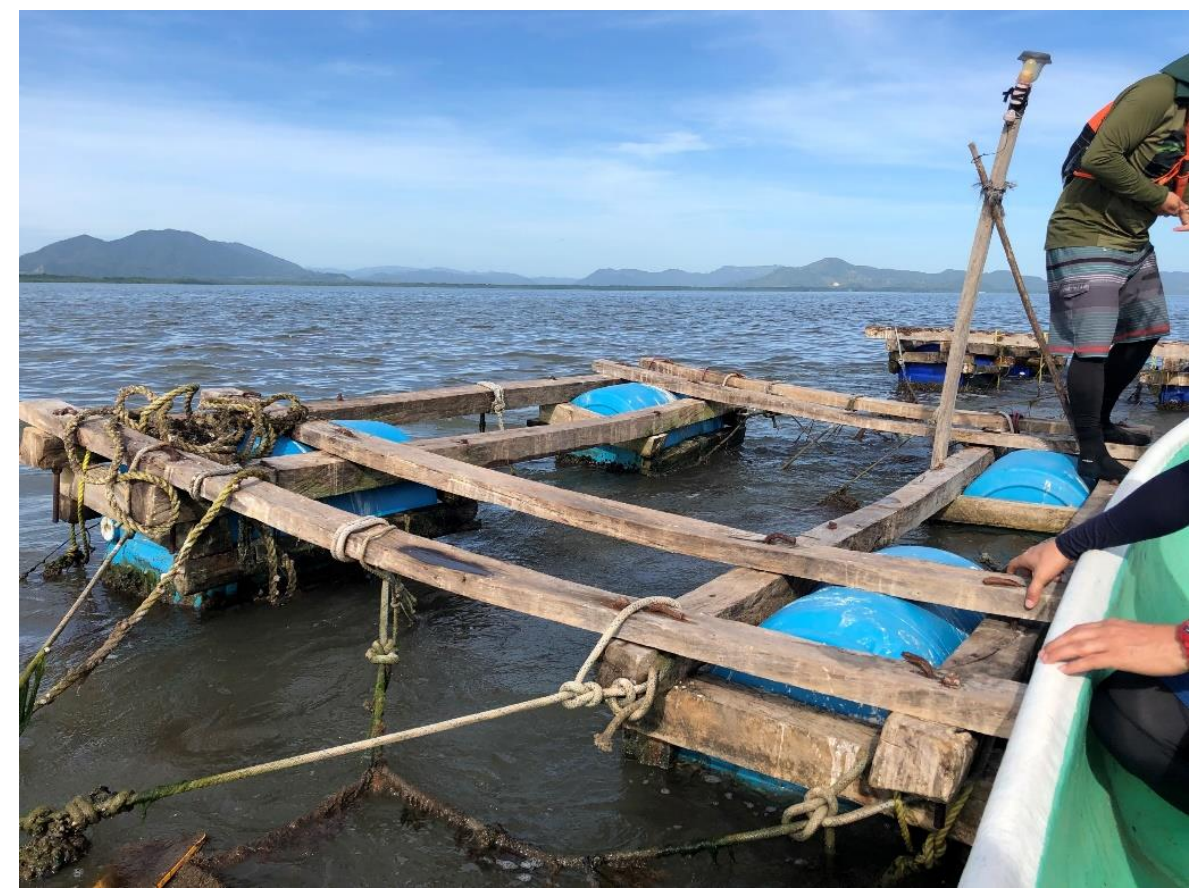

Figura 2. Estructura flotante conocida como "Balsa mejillonera" en Puerto Palito, Isla Chira.

Parámetros medio-ambientales: Los siguientes parámetros físico-quimicos ( $\mathrm{pH}$, salinidad, oxígeno disuelto, temperatura del aire y del agua marina, profundidad) se registraron in situ durante 6 meses. En cada visita al área de estudio, durante 2 días se realizaron 6 muestreos en total (3 muestreos cada día), en los siguientes horarios: 06:00h, 12:00h y 18:00h. Se utilizó para la toma de datos una interfase Vernier Lab Quest 2®, mediante sensores de cada uno de los parámetros anteriormente citados, a excepción de la turbidez, para lo cual se utilizó un Disco secchi. Los datos meteorológicos de precipitación fueron suministrados por el Instituto Meteorológico Nacional de Costa Rica (IMN), mediante la estación "Barco Quebrado, Garza”, ubicada en Nosara, Guanacaste (90 54' 57.77'N, $\left.85^{\circ} 36^{\prime} 52.36^{\prime \prime} \mathrm{W}\right)$.

Mortalidad de organismos: Se seleccionó y se procesó una muestra no probabilística del 10\% de los organismos de la población existente (10 canastas cada mes; $n=60)$. En cada canasta se contabilizó la cantidad total de organismos vivos y muertos, para establecer el porcentaje de mortalidad mensual. Se evaluó la mortalidad producto de las condiciones físico-químicas del medio marino, ya que debido al sello de las canastas se excluyeron a los principales depredadores de la especie. Se aplicó un análisis de correlación de Pearson (r) para determinar una posible incidencia o no de la temperatura del agua marina y la salinidad, sobre la mortalidad del mejillón en el medio de cultivo. Estos análisis se realizaron con un grado de significancia de $\alpha=0,05$ y un $95 \%$ de confiabilidad. 


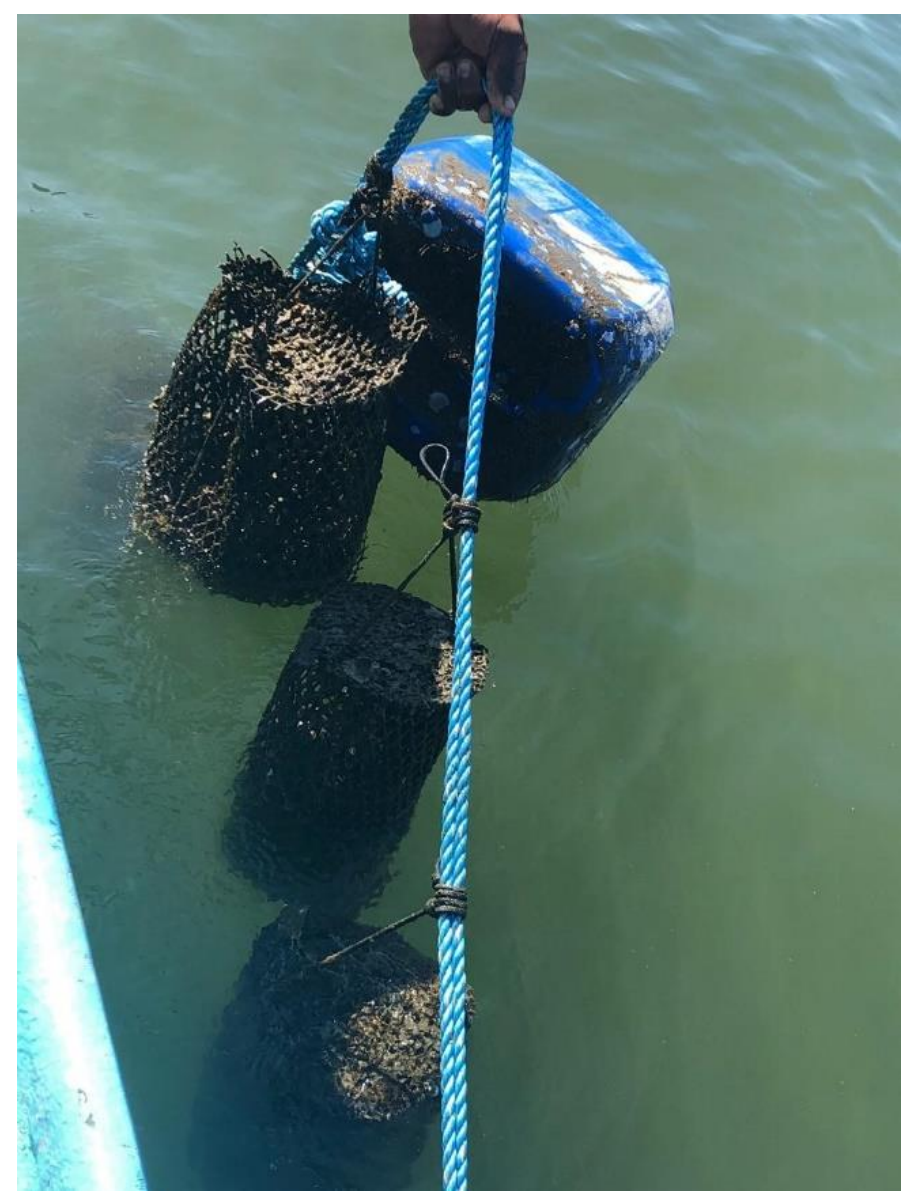

Figura 3. Colectores de semilla y canastas suspendidas con mejillón en el área de cultivo, Palito, Isla Chira.

Crecimiento de organismos: Se seleccionaron y marcaron 5 canastas control con organismos jóvenes de mejillón de aproximadamente de mes de edad (diámetro de la concha $10 \pm 1 \mathrm{~mm}$ ). Cada mes, se midió el largo y el ancho de 10 individuos de cada canasta, para un total de 50 organismos mensuales $(\mathrm{n}=300)$. A estos especímenes se les realizaron biometrías (largo y peso con concha). Se utilizó un "pie de rey" o Vernier digital para las biometrías, mientras que para el pesaje de organismos con concha una balanza digital Sartorius M-prove ${ }^{\circledR}$.

\section{Resultados}

\section{Parámetros ambientales}

Se obtuvieron los siguientes valores de los parámetros ambientales, los cuales se muestran promediados y con sus desviaciones estándar respectivas (ver Cuadro 1). Los criterios y rangos de cada variable en estudio para el cultivo del mejillón se muestran en sección de discusión (Cuadro 2). Los rangos para caracterizar los valores obtenidos fueron tomados de fuentes bibliográficas relacionadas con cultivos de bivalvos y, en algunos casos, específicamente, para el cultivo de mejillón en diferentes regiones del mundo. 
Cuadro 1. Parámetros ambientales en el medio de cultivo de M. guyanensis, Puerto Palito, Isla Chira (Promedio $\pm \mathrm{DE}$ ).

\begin{tabular}{ccccccc}
\hline Parámetros & oct-18 & nov-18 & dic-18 & ene-19 & feb-19 & mar-19 \\
\hline pH & $6,99 \pm 0,40$ & $7,81 \pm 0,28$ & $7,61 \pm 0,32$ & $7,75 \pm 0,41$ & $7,80 \pm 0,43$ & $7,51 \pm 0,99$ \\
\hline $\begin{array}{c}\text { Salinidad (ppm) } \\
\text { Oxígeno disuelto } \\
\text { (mg/L) }\end{array}$ & $3,88 \pm 0,36$ & $4,94 \pm 0,67$ & $4,93 \pm 0,47$ & $5,65 \pm 0,59$ & $4,96 \pm 0,42$ & $3,90 \pm 0,15$ \\
\hline $\begin{array}{c}\text { Temperatura del } \\
\text { aire }\left({ }^{\circ} \mathrm{C}\right)\end{array}$ & $29,75 \pm 5,82$ & $30,16 \pm 5,94$ & $31,02 \pm 4,40$ & $31,36 \pm 4,59$ & $29,07 \pm 5,96$ & $31,07 \pm 4,67$ \\
\hline $\begin{array}{c}\text { Temperatura del } \\
\text { Mar }\left({ }^{\circ} \mathrm{C}\right)\end{array}$ & $28,68 \pm 0,55$ & $30,46 \pm 0,64$ & $30,51 \pm 1,47$ & $29,96 \pm 2,12$ & $28,03 \pm 4,52$ & $31,58 \pm 4,80$ \\
\hline $\begin{array}{c}\text { Turbidez (m) } \\
\text { Profundidad (m) }\end{array}$ & $1,7 \pm 0,20$ & $1,9 \pm 0,19$ & $2,2 \pm 0,29$ & $2 \pm 0,25$ & $2,1 \pm 0,31$ \\
\hline $\begin{array}{c}\text { Precipitación } \\
(\text { mm) }\end{array}$ & 732,3 & $5,05 \pm 0,47$ & $4,91 \pm 0,80$ & $4,98 \pm 0,68$ & $4,86 \pm 0,59$ & $4,40 \pm 0,72$ \\
\hline
\end{tabular}

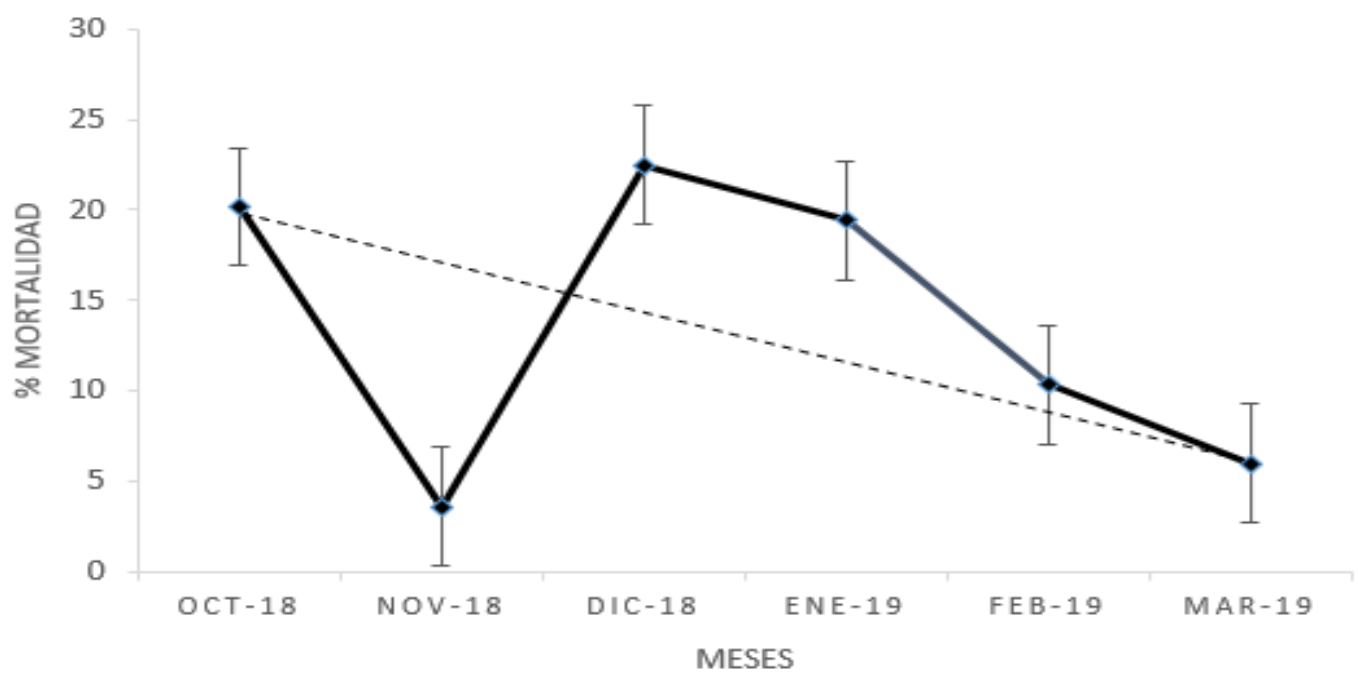

Figura 4. Mortalidad de M. guyanensis en canastas suspendidas, Palito, Isla Chira.

\section{Mortalidad de mejillones en canastas}

Se obtuvo una mortalidad para $M$. guyanensis en promedio de $13,6 \% \pm 4,56$. En Noviembre 2018, se registró la tasa más baja de mortalidad, con tan solo un 3,6 \%; mientras que con un 22,45\% en diciembre 2018, se alcanzó la tasa de mortalidad de organismos más alta (Figura 4). No se logró determinar que la salinidad $(\mathrm{r}=0,35)$, la temperatura ambiental $(\mathrm{r}=0,20)$ o la temperatura del agua $(\mathrm{r}=0,09)$, sean factores que incidan directamente en la mortalidad de esta especie, según los valores promedios obtenidos $(P>0,05)$. 


\section{Crecimiento de mejillones en canastas}

La tasa de crecimiento mensual promedio del largo fue de 0,69 $\pm 0,13 \mathrm{~cm}( \pm \mathrm{DE})$, mientras que el aumento mensual promedio en el peso fue de 1,14 $\pm 1,52 \mathrm{~g}( \pm \mathrm{DE})$. Se logró determinar que, a partir del cuarto mes, mediante este método de cultivo, el mejillón alcanza un tamaño apto para comercialización y consumo ( $\geq 40 \mathrm{~mm}$ de longitud) según la literatura (Pereira, Hilberath, Ansarah y Galvão, 2018) (Figura 5).
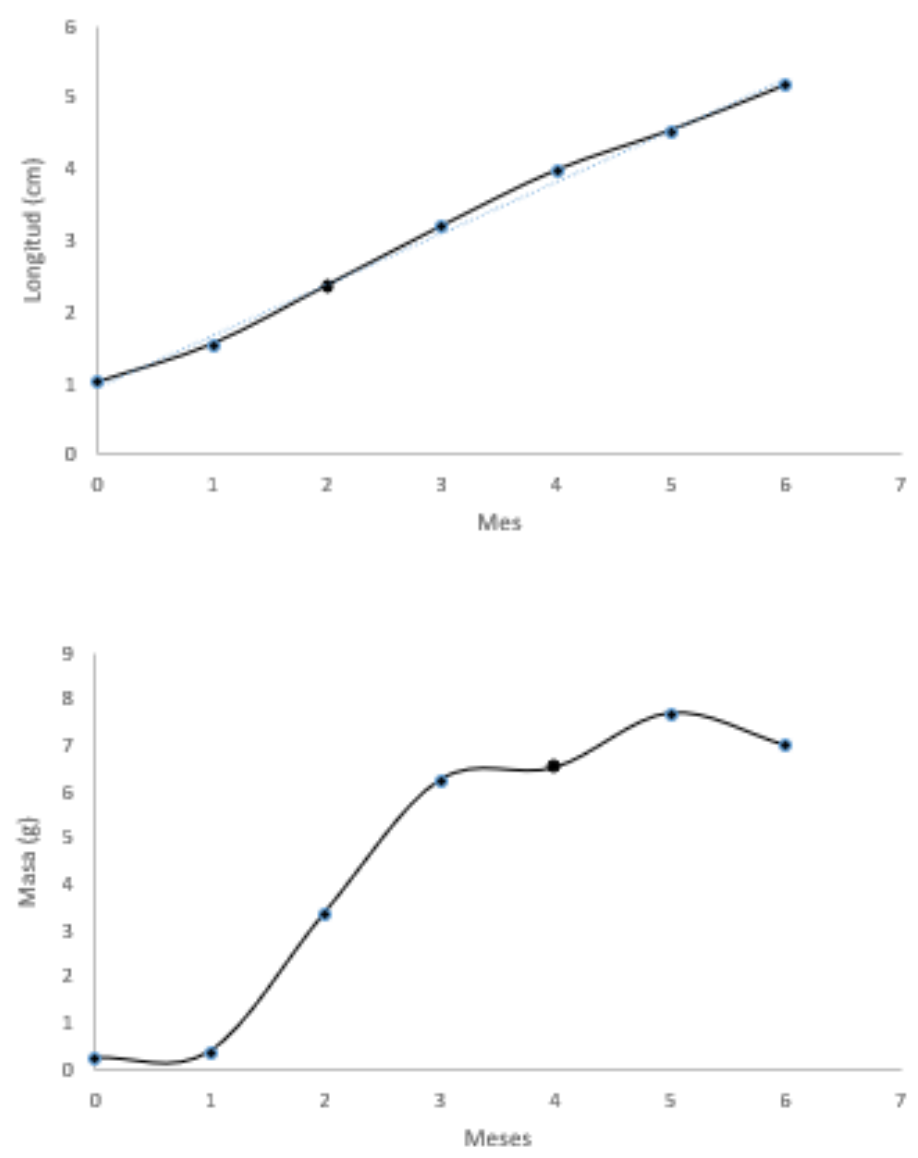

Figura 5. Longitud y peso de M. guyanensis cultivado en canastas suspendidas en Palito, Isla Chira.

\section{Discusión}

\section{Parámetros ambientales}

Una de las limitantes para efectuar comparaciones y determinar rangos óptimos de parámetros físicoquímicos para el cultivo de bivalvos en el área de estudio, es que no se cuenta actualmente con datos a nivel país o región sobre criterios que guíen la producción de estos organismos en proyectos de Maricultura. Destaca el estudio de Quesada (2018) realizado en el Golfo de Nicoya, donde uno de sus puntos de muestreo se realizó en un área cercana a Puerto Palito, Isla Chira. En este, se identificaron sitios óptimos para el cultivo de ostras, considerando algunas de las principales variables ambientales incluidas en el presente trabajo. 
Los parámetros analizados variaron mensualmente, se identificaron tres de ellos como óptimos según la literatura para el cultivo de bivalvos: $\mathrm{pH}$, temperatura del aire y la temperatura del mar (ver Figura 6). Al encontrarse las canastas al menos a dos metros de profundidad, se regula la temperatura del agua a la cual se encuentran, como también se reduce la radiación solar directa a la que podrían estar expuestos, tal como sucede con los organismos en bancos naturales. Se ha logrado determinar que $M$. guyanensis tiene un amplio margen de tolerancia a condiciones ambientales, se ha identificado a la especie como eurihalina (Solomão, Magalhães y Luneta, 1980; Leonel y Silva, 1988), osmoconformista, lo cual se realiza ajustando su volumen celular (Hossoi, Kubota, Toyohara y Hayashi, 2003).

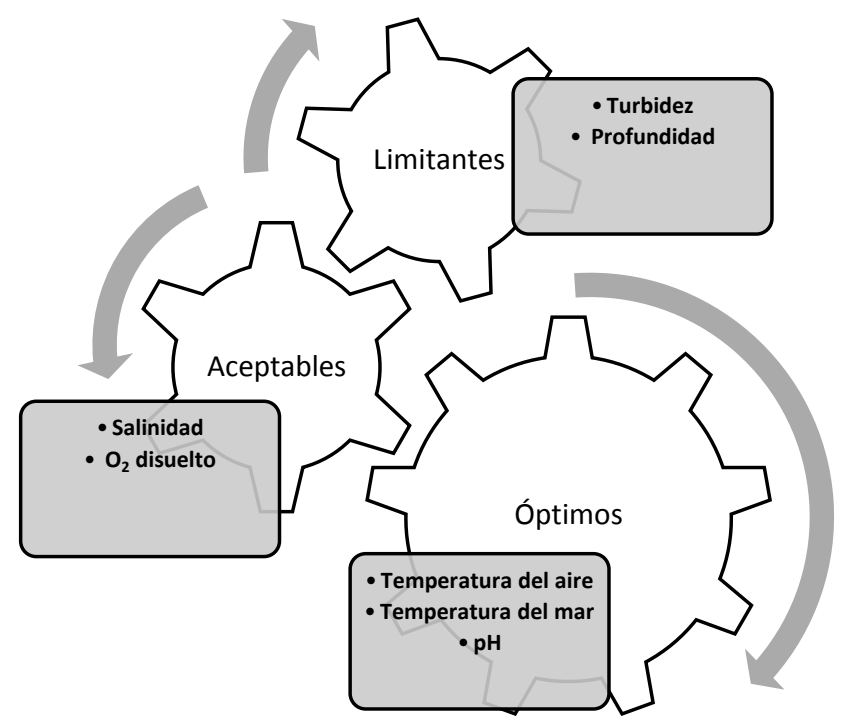

Figura 6. Categorización de parámetros ambientales muestreados, según la literatura especializada en bivalvos.

Únicamente, los parámetros de turbidez y profundidad fueron considerados, según la literatura (Figura 6), como factores limitantes para el cultivo de mejillones en Palito, Isla Chira. Se ha identificado que, para el cultivo del mejillón M. guyanensis en Isla Chira, al menos se necesitaría una claridad en el agua de 0,4m (Bolaños, 1988), lo cual no sería un factor limitante en la actualidad, según los datos obtenidos. Moreno, Quintero y López (2010) identifican que valores por debajo de tres metros de turbidez medibles con disco secchi son considerados sitios de baja calidad para cultivos marinos. Las lluvias y condiciones del viento al final de la época lluviosa suelen arrastrar sedimentos y partículas orgánicas que permanecen en suspensión; sin embargo, las canastas de mejillón, al estar sumergidas, podrían presentar una menor afectación por esta condición.

Recientemente, mediante un estudio de dos especies de mejillón que se cultivan en Chile (una nativa y otra introducida), se determinó que la profundidad de cultivo (2 y $5 \mathrm{~m}$ ) y otras variables ambientales consideradas no son determinantes para el crecimiento de estos organismos. Estas pequeñas 
diferencias, más bien, se atribuyen a características biológicas particulares de cada especie (Díaz, Sobenes y Machino, 2019).

\section{Mortalidad de mejillones en canastas}

La mortalidad de organismos marinos se origina por distintos factores, tales como parámetros ambientales limitantes, contaminación del medio acuático, métodos y técnicas de cultivo inadecuadas, depredación, entre otras. Por ejemplo, los cambios de temperatura y de salinidad en el medio constituyen las principales causas en las tasas de mortalidad de bivalvos (Onodera y Henriques, 2017). Costa y Nalesso (2002) observaron un incremento en la mortalidad de mejillones, principalmente la estación seca, además en los meses de menor nivel de precipitación y con índices de salinidad por encima de los 30 ppm. Lo anterior se determinó en áreas de cultivo en canastas en Brasil para las especies Mytella guyanensis y M. falcata.

Los niveles de contaminación en el medio marino constituyen otro factor que incide en altas tasas de mortalidad en otras latitudes del mundo. En Hong Kong, se han registrado tasas de mortalidad anual de hasta el $>90 \%$ de mejillones verdes (Perna viridis), lo anterior producto de los altos niveles de contaminación del medio acuático principalmente por metales pesados (Cheung, 1993).

En el presente estudio, únicamente se evaluó la mortalidad de $M$. guyanensis bajo el método de cultivo en canastas, y se obtuvo una mortalidad promedio mensual de 13,6\%. En otro estudio similar, se evaluó la supervivencia de $M$. guyanensis mediante dos tipos de cultivo, en un sistema de recirculación del agua. Al final del tiempo de monitoreo (seis meses), el método de cultivo en cuerdas obtuvo un promedio de supervivencia del $39 \%$ (mortalidad $61 \%$ ), mientras que, mediante el sistema de linterna o canastas, únicamente sobrevivió el $31 \%$ de los organismos cosechados, es decir, se alcanzó una mortalidad de hasta el 69 \% (Juárez, 2017).

\section{Crecimiento de mejillones en canastas}

Los índices de crecimiento de mejillón mediante canastas en suspensión en Puerto Palito suelen están por encima del crecimiento de estos Mitílidos en bancos naturales. Así lo constató Sibaja (1985a) en Isla Chira, ya que logró estimar un crecimiento mensual del mejillón (M. guyanensis) de entre $5,07 \mathrm{~mm}$ y 5,27 mm mensual en bancos naturales. Esto podría deberse a que las canastas siempre se encuentran sumergidas en el medio acuático, estos organismos tienen a disposición constantemente alimentos y pueden llegar a filtrar grandes cantidades de agua, tanto en marea alta como baja, no así los mejillones en bancos naturales.

El método de cultivo también es un elemento para considerar cuando se desea tener mayores rendimientos y alcanzar talla comercial de mejillones para su venta. Juárez (2017), durante seis meses, evaluó el crecimiento de $M$. guyanensis mediante dos sistemas de cultivo (long line y canastas). Los organismos cosechados en canastas obtuvieron un mayor crecimiento $(3,55 \pm 0,24 \mathrm{~cm})$ en comparación con los cosechados cuerdas tipo long line $(3,32 \pm 0,22 \mathrm{~cm})$. 


\section{Conclusiones}

En términos generales, el área de cultivo del proyecto de cultivo en mejillón en Puerto Palito, cuenta con condiciones ambientales aptas para la actividad. Únicamente se identificaron dos factores (Turbidez-Profundidad) como limitantes según la literatura. No obstante, estos parámetros, pueden ser contrarrestados con un buen manejo operativo del sistema de cultivo, además de un monitoreo continuo de principales parámetros físico-químicos.

La mortalidad registrada para M. guyanensis bajo este tipo de cultivo se puede considerar como esperada, pues no se logró determinar una correlación de los principales parámetros, como lo son: la salinidad $(\mathrm{r}=0,34)$ o la temperatura del agua marina $(\mathrm{r}=0,09)$ en las tasas de mortalidad registradas. No obstante, la densidad de organismos que se colocan en las canastas $(2 \mathrm{~kg})$, es un factor que podría estar incidiendo negativamente en la superviviencia de los mejillones.

Es a partir del cuarto mes de cultivo, que la especie alcanzó una talla igual o mayor a la recomendada para su comercialización ( $\geq 40 \mathrm{~mm}$ de largo). El crecimiento y aumento de peso de M. guyanensis cultivado en canastas suspendidas tiende a ser más rápido que el crecimiento en su entorno natural, según estudios previos realizados en bancos naturales en Puerto Palito.

\section{Recomendaciones}

Se debe de establecer un protocolo que guie el ordenamiento, identificación y manejo de las balsas mejilloneras y canastas instaladas según la biometría de los organismos. Esto permitirá un mejor control y seguimiento de las existencias de mejillones para su comercialización. La creación e implementación de un plan integral para el manejo de subproductos generados de la producción de mejillones, es una tarea pendiente para una gestión ambiental sostenible del proyecto.

Se recomienda que la producción de bivalvos en Isla Chira se realice únicamente a pequeña escala, debido a las condiciones ambientales (profundidad, corrientes marinas, velocidad del viento, entre otros) entorno a un ecosistema de manglares y su vulnerabilidad. Se debe de valorar la eficiencia de otros métodos de cultivo con respecto a la utilizada actualmente (long line, estacas de fondo, entre otras).

Mediante la aplicación de un análisis microbiológico y químico (en carne) en M. guyanensis, se lograría identificar posibles agentes patógenos y metales pesados respectivamente, que pudiesen estar incidiendo el desarrollo y la mortalidad durante su cultivo en canastas suspendidas. Además, estos análisis son de gran importancia en el sector alimentario, para certificar la calidad y asegurar la inocuidad de este producto. Se debe de contemplar la formulación de un protocolo de muestreo de los principales parámetros físico-químicos de forma autónoma y tecnológica, que pueda ser consultada en tiempo real por parte de cualquier actor participante y público en general. El seguimiento sistemático a largo plazo de las condiciones ambientales es de gran interés científico para contemplar las variaciones diarias, estacionales e interanuales en el transcurso del tiempo; esto generará información más acertada para la toma de decisiones por parte de los actores claves de proyectos de Maricultura en el país. 


\section{Agradecimientos}

La presente investigación se enmarca entorno al proyecto: "Plan piloto para el cultivo del cultivo, mercadeo y comercialización del Mejillón (Mytella guyanensis) en el Golfo de Nicoya” de la UNED, agradezco a los investigadores Rodrigo Méndez, Fiorella Gonzalez y Marlon Salazar, su apertura y recomendaciones para con este trabajo. A la directora del Programa de Laboratorio (Prolab) Mag. Ana Ligia Garro, por las facilidades brindadas para utilización de equipo de muestreo y análisis. A la Vicerrectoría de Investigación de la UNED, especialmente a Ana Ruth Chinchilla, por su apoyo para financiar esta iniciativa. A la comunidad de Puerto Palito, a ASOSAGONY, especialmente a don Freddy y Duber.

\section{Referencias}

Arroyo, D., \& Marín, B. (1998). Crecimiento de Mytella guyanensis (Bivalvia: Mytilidae) en balsas flotantes. San José, Costa Rica. Revista Biología Tropical, 46 (1), 21-26.

Astorga, M., E. Rodríguez \& C. Díaz. (2007). Comparison of minerals and trace element concentration in two mollusks from the Strait of Magellan (Chile). J. Food Company Anual 20:273-279.

Bolaños, J. (1988). Estudio preliminar sobre el comportamiento del mejillón (Mytella guyanensis) en un parque fijo y una estructura flotante en Isla de Chira, Costa Rica. Universidad de Costa Rica.

Carvajal, R. (1969). Fluctuación mensual de larvas y crecimiento del mejillón Perna perna y las condiciones ambientales de la Ensenada de Guatapanare, Estado de Sucre, Venezuela. Boletín Instituto Oceanográfico, 8(1), 13-120.

Cheung, S. (1993). Population dynamics and energy budgets of green-lipped mussel Perna viridis (Linnaeus) in a polluted harbour. Journal of Experimental Marine Biology and Ecology, 168(1), 1-24.

Colombo, J., Varisco, M., Isola, T., Crovetto, C., Rost, E., \& Risso, S. (2016). Composición química proximal y perfil de ácidos grasos del mejillón Mytilidus edulis provenientes de cultivos y bancos naturales en el Golfo San Jorge, Argentina. Revista Biología Marina y Oceanografía, 51(2), 293-299.

Costa, K. \& Nalesso, R. (2002). Cultivo experimental de Mytella falcata y M. guyanensis (Lamarck, 1819) en el estuario de Rio Piraquê-Açu (Aracruz, ES). Acta Limnologia. Brasileira: 14 (1):15-22.

Cruz, R., \& Villalobos, C. (1993a). Monthly changes in tissue weight and biochemical composition of the mussel Mytella guyanensis (Bivalvia: Mytilidae) in Costa Rica. Revista Biología Tropical, 41(1), 93-96.

Cruz, R., \& Villalobos, C. (1993b). Shell lenght at sexual maturity and spawning cycle of Mytella guyanensis (Bivalvia: Mytilidae) from Costa Rica. Revista Biología Tropical, 41(1), 89-92. 
Diarte-Plata, G., Escamilla-Montes, R., De la Cruz-Agüero, G Granados-Alcantar, S., \& ÁlvarezRuiz, P. (2013). Crecimiento y supervivencia del mejillón Mytella strigata (Bivalvia: Mytilidae) en cultivos suspendido en la Laguna Macapule, Sinaloa, México. Revista Hidrobiológica, 23(2), 375-384.

Díaz, C., Sobenes, C., \& Machino, S. (2019). Comparative growth of Mytilus chilensis (Hupé, 1854) and Mytilus galloprovincialis (Lamarck, 1819) in aquaculture longline system in Chile. Aquaculture, 507 (1) 21-27.

FAO. (2018). El estado mundial de la pesca y la acuicultura: Cumplir con los objetivos de desarrollo sostenible.

Fuentes, A., I. Fernández-Segovia, I. Escriche \& J. Serra (2009). Comparison of physicochemical parameters and composition of mussels (Mytilus galloprovincialis) from different Spanish origins. Food Chemistry. 112: 295-302.

Hossoi, M., Kubota, S., Toyohara, H. \& Hayashi, I. (2003). Effect of salinity change on free amino acid content in Pacific oyster. Fisheries Science, 69 (1), 395-400.

Juarez, C. (2017). Comparacion de dos sistemas de cultivo en suspension en el crecimiento y supervivencia de Mytella guyanensis mediante recirculacion del agua. Tesis de Pregrado para obtener el titulo Profesional de Ingeniero Pesquero. Universidad Nacional de Tumbes. Tumbes, Perú.

Leighton, L. (1979). Agrowth prolitic for the rock scallop Hinnites multirugosuls held at several depths off La Jolla, California. Marine Biology, 51(1), 229-332.

Leonel, R. \& Silva, I. (1988). Estudio de sobrevivencia y aislamiento de Mytella guyanensis (Mollusca:Bivalvia) en diferentes salinidades. Revista Nordeste de Biologìa, 6 (1): 35-41.

Mesas, A., \& Tarifeño, E. (2015). Temperaturas letales superiores para el mejillón Mytilus galloprovincialis, en la costa de Chile central. Latin American Journal of Aquatic Research, 43(3), 473-483.

Moreno, D., Quintero, J., \& López, A. (2010). Métodos para identificar, diagnosticar y evaluar el grado de eutrofia. Contactos, 78(1), 25-33.

Onodera, F. \& Henriques, M. (2017). Mortality of Mytella falcata and M. guyanensis exposed to different temperatures. Boletim of Instituto De Pesca, São Paulo, Brasil. 43(1): 106 - 111.

Pereira, O., Hilberath, R., Ansarah, P., \& Galvão, M. (2018). Production estimate of Mytella falcata and M. guyanensis in natural beds of Ilha Comprida estuary (São Paulo, Brasil). Boletim Do Instituto De Pesca, 29 (2), 139-149.

Quesada, R. (2018). Identificación de los sitios óptimos para el cultivo de ostras en el golfo de Nicoya, Costa Rica utilizando los sistemas de información geográfica como insumo para el ordenamiento espacial marino (tesis inédita de maestría). Universidad Nacional de Costa Rica, Heredia, Costa Rica.

Ross, E., Posada, J., Piedra, A., Díaz, J., \& Melo, G. (2014). Guía de identificación: Invertebrados marinos de importancia comercial en la costa Pacífica de Costa Rica. Fundación MarViva. San José, Costa Rica. 
Sibaja, W. G. (1985a). Características biométricas y desarrollo sexual del mejillón Mytella guyanensis (Bivalvia: Mylilidae), en el Litoral Pacífico de Costa Rica (T. de Maestría, Ed.). San José, Costa Rica: Universidad de Costa Rica.

Sibaja, W. G. (1985b). Dimensiones de la concha del mejillón Mytella strigata (Bivalvia: Mollusca), de la playa de Lepanto, Puntarenas, Costa Rica. Revista Biología Tropical, 33(1), 59-60.

Sibaja, W. G. (1986). Madurez sexual en el mejillón chora Mytella guyanensis (Bivalvia: Mylilidae) del manglar en Jicaral, Puntarenas, Costa Rica. Revista Biología Tropical, 34(1), 151-155.

Solomão, L., Magalhães, A. \& Lunetta, J. (1980). Influencia de la salinidad en la sobrevivencia de Perna perna (Mollusca:Bivalvia). Boletin de fisiologia Animal Universidad de Sãn Paulo, 4 (1) 143-152.

Timmons, M., Ebeling, J. \& Piedrahita, R. (2009). Acuicultura en Sistemas de Recirculación. Cayuga Aqua Ventures. NRACE Publication No. 101-109 Spanish. 